\title{
Ehrhart series of fractional stable set poly- topes of finite graphs
}

\author{
Ginji Hamano, Takayuki Hibi and Hidefumi Ohsugi
}

\begin{abstract}
The fractional stable set polytope $\operatorname{FRAC}(G)$ of a simple graph $G$ with $d$ vertices is a rational polytope that is the set of nonnegative vectors $\left(x_{1}, \ldots, x_{d}\right)$ satisfying $x_{i}+x_{j} \leq 1$ for every edge $(i, j)$ of $G$. In this paper we show that (i) The $\delta$-vector of a lattice polytope $2 \operatorname{FRAC}(G)$ is alternatingly increasing, (ii) The Ehrhart ring of $\operatorname{FRAC}(G)$ is Gorenstein, (iii) The coefficients of the numerator of the Ehrhart series of $\operatorname{FRAC}(G)$ are symmetric, unimodal and computed by the $\delta$-vector of $2 \operatorname{FRAC}(G)$.
\end{abstract}

Mathematics Subject Classification (2010). Primary 52B05; Secondary $52 \mathrm{~B} 20$.

Keywords. Ehrhart series, Ehrhart rings, fractional stable set polytopes, Gorenstein Fano polytopes, unimodal $\delta$-vectors.

\section{Introduction}

The Ehrhart series of a rational convex polytope is one of the most important topics in combinatorics. Let $P$ be a $d$-dimensional rational convex polytope in $\mathbb{R}^{N}$. For each $n \in \mathbb{N}$, let $n P=\{n \alpha \mid \alpha \in P\}$ and define the function $i(P, n):=\sharp\left(n P \cap \mathbb{Z}^{N}\right)$. Thus $i(P, n)$ is the number of lattice points contained in $n P$, called the Ehrhart quasi-polynomial of $P$. It is known that $i(P, n)$ is indeed a quasi-polynomial of degree $d$. In particular, if $P$ is a lattice polytope, i.e., all vertices of $P$ are lattice points, then $i(P, n)$ is a polynomial and called the Ehrhart polynomial of $P$. The generating function of the Ehrhart quasi-polynomial is defined by $E(P, t):=1+\sum_{n=1}^{\infty} i(P, n) t^{n}$ and called the Ehrhart series of $P$. Let $m$ be the smallest natural number $k$ for which $k P$ is a lattice polytope and let $v$ be the smallest natural number $k$ for which $k P$ has a lattice point in its interior. It is known that $E(P, t)$ is a rational function of degree $-v$ and has an expression $E(P, t):=g(P, t) /\left(1-t^{m}\right)^{d+1}$ where $g(P, t)$ is a polynomial of degree $m(d+1)-v$ with nonnegative integer coefficients. In particular, if $P$ is a lattice polytope, then $m=1$ and hence 
$E(P, t)=\delta(P, t) /(1-t)^{d+1}$ where $\delta(P, t)=\delta_{0}+\delta_{1} t+\cdots+\delta_{d+1-v} t^{d+1-v}$ is a polynomial of degree $d+1-v$, called the $\delta$-polynomial of $P$. The coefficients $\left(\delta_{0}, \ldots, \delta_{d+1-v}\right)$ of $\delta(P, t)$ is called the $\delta$-vector (or $h$-vector, $h^{*}$-vector) of $P$. Next, we define the Ehrhart ring of a $d$-dimensional rational polytope $P \subset \mathbb{R}^{N}$. Let $\left[A_{K}(P)\right]_{n}$ be the linear space over a field $K$ whose basis is the set of Laurent monomials $x_{1}^{\alpha_{1}} \cdots x_{N}^{\alpha_{N}} t^{n}$ with $\left(\alpha_{1}, \ldots, \alpha_{N}\right) \in n P \cap \mathbb{Z}^{N}$. Then $A_{K}(P):=\bigoplus_{n \geq 0}\left[A_{K}(P)\right]_{n}$ is called the Ehrhart ring of $P$. If $A_{K}(P)$ is Gorenstein, then the coefficients of the numerator of $E(P, t)$ are symmetric. The dual polytope of $P$ is defined by $P^{\vee}=\left\{\mathbf{x} \in \mathbb{R}^{d} \mid\langle\mathbf{x}, \mathbf{y}\rangle \leq 1\right.$ for all $\left.\mathbf{y} \in P\right\}$, where $\langle\mathbf{x}, \mathbf{y}\rangle$ is the usual inner product of $\mathbb{R}^{d}$. The notion of dual polytopes appears in a criterion for $A_{K}(P)$ to be Gorenstein. Let $P \subset \mathbb{R}^{d}$ be a lattice polytope of dimension $d$. We say that $P$ is a Fano polytope if the origin of $\mathbb{R}^{d}$ is the unique lattice point belonging to the interior of $P$. A Fano polytope is called Gorenstein if its dual polytope is a lattice polytope. (A Gorenstein Fano polytope is often called a reflexive polytope in the literature.)

Let $G$ be a finite simple graph on the vertex set $[d]=\{1,2, \ldots, d\}$ and let $E(G)$ be the edge set of $G$. Throughout this paper, we always assume that $G$ has no isolated vertices. Given a subset $W \subset[d]$, we associate the $(0,1)$-vector $\rho(W)=\sum_{j \in W} \mathbf{e}_{j} \in \mathbb{R}^{d}$. Here, $\mathbf{e}_{i}$ is the $i$ th unit coordinate vector of $\mathbb{R}^{d}$. In particular, $\rho(\emptyset)$ is the origin of $\mathbb{R}^{d}$. A subset $W$ is called stable if $\{i, j\} \notin E(G)$ for all $i, j \in W$ with $i \neq j$. Note that the empty set and each single-element subset of $[d]$ are stable. Let $S(G)$ denote the set of all stable sets of $G$. The stable set polytope (independent set polytope) $\operatorname{STAB}(G) \subset \mathbb{R}^{d}$ of a simple graph $G$ is the $(0,1)$-polytope which is the convex full of $\{\rho(W) \mid W \in S(G)\}$. Stable set polytopes are very important in many areas, e.g., optimization theory. The $\delta$-vector of the stable set polytope of a perfect graph is studied in 1, 7]. On the other hand, the fractional stable set polytope $\operatorname{FRAC}(G)$ of $G$ is the $d$-polytope in $\mathbb{R}^{d}$ defined by

$$
\operatorname{FRAC}(G):=\left\{\left(x_{1}, \ldots, x_{d}\right) \in \mathbb{R}^{d} \mid \begin{array}{cc}
x_{i} \geq 0 & (1 \leq i \leq d) \\
x_{i}+x_{j} \leq 1 & ((i, j) \in E(G))
\end{array}\right\} .
$$

In general, we have $\operatorname{STAB}(G) \subset \operatorname{FRAC}(G)$. Each vertex of $\operatorname{FRAC}(G)$ belongs to $\{0,1 / 2,1\}^{d}$ (see, e.g, [6]). It is known that $\operatorname{FRAC}(G)=\operatorname{STAB}(G)$ if and only if $G$ is bipartite. If $G$ is bipartite, then $\operatorname{STAB}(G)$ has a unimodular triangulation, and the $\delta$-vector of $\operatorname{STAB}(G)$ is symmetric and unimodal (see [1, 3, 7]). Note that, if $G$ is bipartite, then $\operatorname{STAB}(G)$ is the chain polytope of a poset $P$ of rank 1 whose comparability graph is $G$, and affinely equivalent to the order polytope of the poset $P$ (see [9]). The purpose of this paper is to study the Ehrhart series of $\operatorname{FRAC}(G)$. The following two polytopes will play important roles:

$$
\begin{aligned}
\mathcal{P}(G) & =2 \cdot \operatorname{FRAC}(G) \\
\mathcal{Q}(G) & =3 \cdot \operatorname{FRAC}(G)-(1, \ldots, 1) \\
& =\left\{\left(x_{1}, \ldots, x_{d}\right) \in \mathbb{R}^{d} \mid \begin{array}{cc}
x_{i} \geq-1 & (1 \leq i \leq d) \\
x_{i}+x_{j} \leq 1 & ((i, j) \in E(G))
\end{array}\right\} .
\end{aligned}
$$


In [13, Steingrímsson called the lattice polytope $\mathcal{P}(G)$ the extended 2-weak vertex-packing polytope of $G$ and studied the structure of $\mathcal{P}(G)$. In particular, he constructed a unimodular triangulation of $\mathcal{P}(G)$ and showed that the $\delta$-vector of $\mathcal{P}(G)$ is obtained by a descent statistic on a subset of the hyperoctahedral group determined by $G$.

This paper is organized as follows. In Section 1, we show that the $\delta$ vector $\left(\delta_{0}, \ldots, \delta_{d-1}\right)$ of $\mathcal{P}(G)$ is alternatingly increasing ([10, Definition 2.9]), i.e.,

$$
\delta_{0} \leq \delta_{d-1} \leq \delta_{1} \leq \delta_{d-2} \leq \cdots \leq \delta_{\lfloor d / 2\rfloor-1} \leq \delta_{d-\lfloor d / 2\rfloor} \leq \delta_{\lfloor d / 2\rfloor} .
$$

In Section 2, we study the structure of $\mathcal{Q}(G)$ in order to show that the Ehrhart ring of $\operatorname{FRAC}(G)$ is Gorenstein. By using this result, in Section 3, we give a formula for the numerator of the Ehrhart series $E(\operatorname{FRAC}(G), t):=$ $g(\operatorname{FRAC}(G), t) /\left(1-t^{2}\right)^{d+1}$ via the $\delta$-vector of $\mathcal{P}(G)$. Since the Ehrhart ring of $\operatorname{FRAC}(G)$ is Gorenstein and since the $\delta$-vector of $\mathcal{P}(G)$ is alternatingly increasing, it follows that the coefficients of $g(\operatorname{FRAC}(G), t)$ is symmetric and unimodal. Finally, in Section 4, we discuss the dual polytope $\mathcal{Q}(G)^{\vee}$ of $\mathcal{Q}(G)$.

\section{The $\delta$-vector of $\mathcal{P}(G)$}

First, we review the results in [13]. Let $B_{d}$ denote the all signed permutation words on $[d]=\{1,2, \ldots, d\}$. For example, if $d=2$,

$$
B_{2}=\{12,21, \overline{1} 2,2 \overline{1}, 1 \overline{2}, \overline{2} 1, \overline{1} \overline{2}, \overline{2} \overline{1}\},
$$

where $\overline{1}=-1$ and $\overline{2}=-2$. We order the letters in signed permutations as integers, i.e., $\cdots<\overline{3}<\overline{2}<\overline{1}<0<1<2<3<\cdots$. An element $i \in[d]$ is called a descent in $\pi=a_{1} \cdots a_{d} \in B_{d}$ if one of the following holds ([13, Definition 5]):

(i) $i<d$ and $a_{i}>a_{i+1}$;

(ii) $i=d$ and $a_{i}>0$.

Let $\operatorname{des}(\pi)$ denote the number of descents in $\pi \in B_{d}$. For example, for $\pi=$ $2 \overline{3} \overline{4} 1 \in B_{4}, \operatorname{des}(\pi)=3$ since the descents of $\pi$ are 1, 2 and 4 . For any subset $S$ of $B_{d}$, the descent polynomial of $S$ is $D(S, t):=\sum_{\pi \in S} t^{\operatorname{des}(\pi)}$. Let $G$ be a simple graph on the vertex set $[d]$ and the edge set $E(G)$. We define a subset $\Pi(G)$ of $B_{d}$ as follows ([13, Definition 11 and Theorem 12]):

$$
\Pi(G)=\left\{\begin{array}{l|l}
\pi \in B_{d} & \begin{array}{c}
\text { if }(i, j) \in E(G) \text { and }+i \text { appears in } \pi \\
\text { then }-j \text { must precede }+i \text { in } \pi
\end{array}
\end{array}\right\} .
$$

Proposition 1.1 ([13]). Let $G$ be a finite simple graph. Then the $\delta$-polynomial of $\mathcal{P}(G)$ equals the descent polynomial $D(\Pi(G), t)$.

By using this fact, we will show Theorem 1.2 below. Note that a similar decomposition technique (i.e., $a(t)+t b(t))$ was used in 12 to establish Ehrhart inequalities originally due to Stanley and Hibi. 
Theorem 1.2. Let $G$ be a simple graph with $d$ vertices. Then there exist symmetric and unimodal polynomials $a(t)$ of degree $d-1$ and $b(t)$ of degree $d-2$ such that $\delta(\mathcal{P}(G), t)=a(t)+t b(t)$. In particular, the $\delta$-vector $\left(\delta_{0}, \delta_{1}, \ldots, \delta_{d-1}\right)$ of $\mathcal{P}(G)$ is alternatingly increasing, i.e.,

$$
\delta_{0} \leq \delta_{d-1} \leq \delta_{1} \leq \delta_{d-2} \leq \cdots \leq \delta_{\lfloor d / 2\rfloor-1} \leq \delta_{d-\lfloor d / 2\rfloor} \leq \delta_{\lfloor d / 2\rfloor} .
$$

Proof. Let $\Pi_{+}$(resp. $\Pi_{-}$) denote the set of all $\pi \in \Pi(G)$ such that the last number of $\pi$ is positive (resp. negative). Note that the first number of $\pi \in \Pi(G)$ is always negative since $G$ has no isolated vertices.

Let $\pi \in \Pi_{+}$. Then $\pi$ has a representation

$$
\pi=m_{1}^{(1)} \cdots m_{\alpha_{1}}^{(1)} p_{1}^{(1)} \cdots p_{\beta_{1}}^{(1)} m_{1}^{(2)} \cdots m_{\alpha_{2}}^{(2)} p_{1}^{(2)} \cdots p_{\beta_{2}}^{(2)} \cdots m_{1}^{(\gamma)} \cdots m_{\alpha_{\gamma}}^{(\gamma)} p_{1}^{(\gamma)} \cdots p_{\beta_{\gamma}}^{(\gamma)}
$$

where $p_{i}^{(j)}>0$ and $m_{i}^{(j)}<0$. Let $S(\pi)$ denote the set of all signed permutation words on $[d]$ of the form

$$
m_{\sigma_{1}(1)}^{(1)} \cdots m_{\sigma_{1}\left(\alpha_{1}\right)}^{(1)} p_{\tau_{1}(1)}^{(1)} \cdots p_{\tau_{1}\left(\beta_{1}\right)}^{(1)} \cdots m_{\sigma_{\gamma}(1)}^{(\gamma)} \cdots m_{\sigma_{\gamma}\left(\alpha_{\gamma}\right)}^{(\gamma)} p_{\tau_{\gamma}(1)}^{(\gamma)} \cdots p_{\tau_{\gamma}\left(\beta_{\gamma}\right)}^{(\gamma)}
$$

where $\sigma_{k} \in \mathcal{S}_{\alpha_{k}}$ and $\tau_{k} \in \mathcal{S}_{\beta_{k}}$ are permutations. It is easy to see that $S(\pi) \subset$ $\Pi_{+}$. Let $A_{k}(t)=\sum_{i=0}^{k-1} A(k, i) t^{i}$ denote the Eulerian polynomial whose coefficients $A(k, i)$ is Eulerian number. It is known that $A_{k}(t)=\sum_{\pi \in \mathcal{S}_{k}} t^{d(\pi)}$, where $d(\pi)$ is the number of usual descent of $\pi$ (i.e., no signs involved and never a descent at $k$ ). See, e.g., [8]. Thus

$$
D(S(\pi), t)=t^{\gamma} \prod_{j=1}^{\gamma} A_{\alpha_{j}}(t) A_{\beta_{j}}(t) .
$$

It is known that $(A(k, 0), A(k, 1), \ldots, A(k, k-1))$ is symmetric and unimodal, i.e., $A(k, i) \leq A(k, i+1)$ for $0 \leq i \leq\lfloor 2 / k\rfloor$. The degree of $D(S(\pi), t)$ is $\gamma+\sum_{j=1}^{\gamma}\left(\alpha_{j}+\beta_{j}-2\right)=d-\gamma$. Since $A_{k}(t)$ is symmetric and unimodal, so is $A_{\alpha_{j}}(t) A_{\beta_{j}}(t)$. Hence

$$
D(S(\pi), t)=s_{\gamma} t^{\gamma}+\cdots+s_{d-\gamma} t^{d-\gamma}
$$

satisfies that $\left(s_{\gamma}, \ldots, s_{d-\gamma}\right)$ is symmetric and unimodal. Since

$$
D\left(\Pi_{+}, t\right)=u_{1} t+\cdots+u_{d-1} t^{d-1}
$$

is a sum of such $D(S(\pi), t)^{\prime}$ 's, $\left(u_{1}, \ldots, u_{d-1}\right)$ is symmetric and unimodal.

Let $\pi \in \Pi_{-}$. Then $\pi$ has a representation

$$
\pi=m_{1}^{(1)} \cdots m_{\alpha_{1}}^{(1)} p_{1}^{(1)} \cdots p_{\beta_{1}}^{(1)} m_{1}^{(2)} \cdots m_{\alpha_{2}}^{(2)} p_{1}^{(2)} \cdots p_{\beta_{2}}^{(2)} \cdots m_{1}^{(\gamma)} \cdots m_{\alpha_{\gamma}}^{(\gamma)},
$$

where $p_{i}^{(j)}>0$ and $m_{i}^{(j)}<0$. Define $S(\pi)$ as before. Then we have

$$
D(S(\pi), t)=t^{\gamma-1} A_{\alpha_{\gamma}}(t) \prod_{j=1}^{\gamma-1} A_{\alpha_{j}}(t) A_{\beta_{j}}(t) .
$$

The degree of $D(S(\pi), t)$ is $\gamma-1+\alpha_{\gamma}-1+\sum_{j=1}^{\gamma-1}\left(\alpha_{j}+\beta_{j}-2\right)=d-\gamma$. Since

$$
D\left(\Pi_{-}, t\right)=v_{0}+v_{1} t+\cdots+v_{d-1} t^{d-1}
$$


is a sum of such $D(S(\pi), t)$ 's, $\left(v_{0}, v_{1}, \ldots, v_{d-1}\right)$ is symmetric and unimodal.

We now show that the $\delta$-vector $\left(\delta_{0}, \ldots, \delta_{d-1}\right)=\left(v_{0}, u_{1}+v_{1}, \ldots, u_{d-1}+\right.$ $\left.v_{d-1}\right)$ of $\mathcal{P}(G)$ is alternatingly increasing. First, $\delta_{d-1}-\delta_{0}=u_{d-1}+v_{d-1}-$ $v_{0}=u_{d-1} \geq 0$. Moreover, for $i=1,2, \ldots,\lfloor d / 2\rfloor$, we have $\delta_{i}-\delta_{d-i}=u_{i}+$ $v_{i}-u_{d-i}-v_{d-i}=v_{i}-v_{i-1} \geq 0$, and for $i=1,2, \ldots,\lfloor d / 2\rfloor-1$, we have $\delta_{d-i-1}-\delta_{i}=u_{d-i-1}+v_{d-i-1}-u_{i}-v_{i}=u_{i+1}-u_{i} \geq 0$. Thus the $\delta$-vector of $\mathcal{P}(G)$ is alternatingly increasing.

\section{The Ehrhart ring of $\operatorname{FRAC}(G)$}

In this section, we will show that the Ehrhart ring of $\mathrm{FRAC}(G)$ is Gorenstein. In order to show that the Ehrhart ring of $\operatorname{FRAC}(G)$ is Gorenstein, we will use the following criterion [5, Theorem 1.1]:

Proposition 2.1. Let $P \subset \mathbb{R}^{d}$ be a rational convex polytope of dimension $d$ and let $\delta \geq 1$ denote the smallest integer for which $\delta(P \backslash \partial P) \cap \mathbb{Z}^{d} \neq \emptyset$. Fix $\alpha \in \delta(P \backslash \partial P) \cap \mathbb{Z}^{d}$ and let $Q=\delta P-\alpha \subset \mathbb{R}^{d}$. Then the Ehrhart ring $A_{K}(P)$ of $P$ is Gorenstein if and only if the following conditions are satisfied:

(i) The dual polytope $Q^{\vee}$ of $Q$ is a lattice polytope;

(ii) Let $\widetilde{P} \subset \mathbb{R}^{d+1}$ denote the rational convex polytope which is the convex hull of the subset $\left\{(\beta, 0) \in \mathbb{R}^{d+1} \mid \beta \in P\right\} \cup\{(0, \ldots, 0,1 / \delta)\}$ in $\mathbb{R}^{d+1}$. Then $\widetilde{P}$ is facet-reticular, that is to say, if $H$ is a hyperplane in $\mathbb{R}^{d+1}$ and if $H \cap \widetilde{P}$ is a facet of $\widetilde{P}$, then $H \cap \mathbb{Z}^{d+1} \neq \emptyset$.

It is clear that there exists no lattice points in the interior of $\mathcal{P}(G)=$ $2 \mathrm{FRAC}(G)$, and that the lattice point $(1, \ldots, 1)$ belongs to the interior of 3 FRAC $(G)$. Thus it is enough to show that conditions (i) and (ii) in Proposition 2.1 are satisfied when $P=\operatorname{FRAC}(G), \delta=3, \alpha=(1, \ldots, 1)$ and $Q=\mathcal{Q}(G)$. A criterion for a vector to be a vertex of $\operatorname{FRAC}(G)$ is given in [13, Theorem 15]:

Lemma 2.2. Let $G$ be a finite simple graph with $d$ vertices. Suppose that $\mathbf{v}=\left(v_{1}, \ldots, v_{d}\right) \in\{0,1 / 2,1\}^{d}$ belongs to $\operatorname{FRAC}(G)$. Let $G_{S}$ be the subgraph of $G$ induced by $S=\left\{i \in[d] \mid v_{i}=1 / 2\right\}$. Then $\mathbf{v}$ is a vertex of $\operatorname{FRAC}(G)$ if and only if either $S=\emptyset$ or each connected component of $G_{S}$ contains an odd cycle.

Using Lemma 2.2, we determine when $\mathcal{Q}(G)$ is a lattice polytope.

Proposition 2.3. Let $G$ be a finite simple graph without isolated vertices. Then the following conditions are equivalent.

(i) The graph $G$ is a bipartite graph;

(ii) The polytope $\operatorname{FRAC}(G)$ is a lattice polytope;

(iii) The polytope $\mathcal{Q}(G)$ is a lattice polytope.

Proof. If $G$ is bipartite, then $\operatorname{FRAC}(G)=\operatorname{STAB}(G)$ is a lattice polytope. Hence (i) $\Rightarrow$ (ii) holds. Moreover, (ii) $\Rightarrow$ (iii) is trivial. We now show that (iii) $\Rightarrow$ (i). Suppose $G$ contains an odd cycle $C$. Let $H$ be a connected component 
of $G$ that contains $C$ and let $V(H)$ be the set of vertices of $H$. Here, we define $\mathbf{v}=\left(v_{1}, \ldots, v_{d}\right)$ by $v_{i}=1 / 2$ if $i \in V(H)$ and $v_{i}=0$ if $i \notin V(H)$. Then $\mathbf{v}$ is a $(0,1 / 2)$-vector in $\operatorname{FRAC}(G)$. Moreover, since $\mathbf{v}$ satisfies the condition in Lemma 2.2, $\mathbf{v}$ is a vertex of $\operatorname{FRAC}(G)$. Then $3 \mathbf{v}-(1, \ldots, 1) \in\{-1,1 / 2\}^{d}$ is a vertex of $\mathcal{Q}(G)$ that is not a lattice point. Hence $\mathcal{Q}(G)$ is not a lattice polytope.

Next we show that $\mathcal{Q}(G)^{\vee}$ is a lattice polytope.

Proposition 2.4. Suppose $G$ is a finite simple graph without isolated vertices. Then the origin of $\mathbb{R}^{d}$ is a unique lattice point belonging to the interior of $\mathcal{Q}(G)$ and

$$
\left\{\mathbf{e}_{i}+\mathbf{e}_{j} \mid(i, j) \in E(G)\right\} \cup\left\{-\mathbf{e}_{i} \mid 1 \leq i \leq d\right\}
$$

is the vertex set of $\mathcal{Q}(G)^{\vee}$. In particular, if $G$ is a bipartite graph, then $\mathcal{Q}(G)$ is a Gorenstein Fano polytope.

Proof. It is known that the inequalities $x_{i} \geq 0(1 \leq i \leq d)$ and $x_{i}+x_{j} \leq 1$ $((i, j) \in E(G))$ define the facets of $\operatorname{FRAC}(G)$. Hence the inequalities $x_{i} \geq-1$ $(1 \leq i \leq d)$ and $x_{i}+x_{j} \leq 1((i, j) \in E(G))$ define the facets of $\mathcal{Q}(G)$. Thus a vector $\left(v_{1}, \ldots, v_{d}\right) \in \mathbb{R}^{d}$ belongs to the interior of $\mathcal{Q}(G)$ if and only if $v_{i}>-1$ $(1 \leq i \leq d)$ and $v_{i}+v_{j}<1((i, j) \in E(G))$. It is clear that the origin of $\mathbb{R}^{d}$ belongs to the interior of $\mathcal{Q}(G)$. Suppose that $\left(v_{1}, \ldots, v_{d}\right) \in \mathbb{Z}^{d}$ belongs to the interior of $\mathcal{Q}(G)$. Since $v_{i}$ and $v_{i}+v_{j}$ are integers, we have $v_{i} \geq 0(1 \leq i \leq d)$ and $v_{i}+v_{j} \leq 0((i, j) \in E(G))$. Hence $v_{i}=0$ for all $i$, i.e, $\left(v_{1}, \ldots, v_{d}\right)=\mathbf{0}$. It is known that there is a one-to-one correspondence between the facets of $\mathcal{Q}(G)$ and the vertices of $\mathcal{Q}(G)^{\vee}$. The set $\left\{\mathbf{e}_{i}+\mathbf{e}_{j} \mid(i, j) \in E(G)\right\} \cup\left\{-\mathbf{e}_{i} \mid 1 \leq i \leq d\right\}$ of coefficient vectors of inequalities that define facets is the set of vertices of $\mathcal{Q}(G)^{\vee}$. Thus, in particular, $\mathcal{Q}(G)^{\vee}$ is a lattice polytope. By Proposition 2.3, if $G$ is a bipartite graph, then $\mathcal{Q}(G)$ is a lattice polytope, and hence a Gorenstein Fano polytope.

We are now in the position to show that the Ehrhart ring of $\operatorname{FRAC}(G)$ is Gorenstein.

Theorem 2.5. Let $G$ be a finite simple graph without isolated vertices. Then the Ehrhart ring of $\operatorname{FRAC}(G)$ is Gorenstein.

Proof. It is enough to show that conditions (i) and (ii) in Proposition 2.1 are satisfied when $P=\operatorname{FRAC}(G), \delta=3, \alpha=(1, \ldots, 1)$ and $Q=\mathcal{Q}(G)$. First, Proposition 2.4 guarantees that $Q^{\vee}$ is a lattice polytope. Let $F=H \cap \widetilde{P}$ be a facet of $\widetilde{P}$, where $H$ is a hyperplane in $\mathbb{R}^{d+1}$. We may assume that $H \neq$ $\left\{x_{d+1}=0\right\}$. Then $F^{\prime}=F \cap\left\{x_{d+1}=0\right\}$ is a facet of $\left\{(\beta, 0) \in \mathbb{R}^{d+1} \mid \beta \in P\right\}$ whose supporting hyperplane is $H$. Therefore, $H^{\prime}=H \cap\left\{x_{d+1}=0\right\}$ is defined by $\left\{x_{d+1}=0\right\}$ and either $x_{i}+x_{j}=1((i, j) \in E(G))$ or $x_{i}=0(1 \leq i \leq d)$. Hence it is clear that there exists a lattice point in $H^{\prime} \subset H$. Thus condition (ii) in Proposition 2.1 holds. Therefore, the Ehrhart ring of $P$ is Gorenstein by Proposition 2.1. 


\section{The Ehrhart series of $\operatorname{FRAC}(G)$}

In this section, we show that we can calculate the Ehrhart series and the Ehrhart quasi-polynomial of $\operatorname{FRAC}(G)$ from that of $\mathcal{P}(G)$. Let $G$ be a simple graph on the vertex set $[d]$ without isolated vertices. Since the interior of $\mathcal{P}(G)$ possesses no lattice points, and the interior of $2 \mathcal{P}(G)$ has a lattice point, it follows that $\operatorname{deg} \delta(\mathcal{P}(G), t)=d+1-2=d-1$. On the other hand, the degree of $E(\operatorname{FRAC}(G), t)$ is -3 as a rational function. Given a rational convex polytope $\mathcal{P}$, the period of $i(\mathcal{P}, n)$ is a divisor of the smallest positive integer $\alpha$ for which $\alpha \mathcal{P}$ is a lattice polytope. See [9, Theorem 4.6.25]. Hence $i(\operatorname{FRAC}(G), n)$ is a quasi-polynomial of period at most 2 . Thus there exist polynomials $i^{\text {odd }}(\operatorname{FRAC}(G), n)$ and $i^{\text {even }}(\operatorname{FRAC}(G), n)$ of degree $d$ such that

$$
i(\operatorname{FRAC}(G), n)= \begin{cases}i^{\text {odd }}(\operatorname{FRAC}(G), n) & \text { if } n \text { is odd, } \\ i^{\text {even }}(\operatorname{FRAC}(G), n) & \text { if } n \text { is even. }\end{cases}
$$

In particular, if $G$ is bipartite, then $i^{\text {odd }}(\operatorname{FRAC}(G), n)=i^{\operatorname{even}}(\operatorname{FRAC}(G), n)$.

Theorem 3.1. Let $G$ be a simple graph on the vertex set $[d]$ without isolated vertices and let $\delta(\mathcal{P}(G), t)=\delta_{0}+\delta_{1} t+\cdots+\delta_{d-1} t^{d-1}$. Then we have

$$
\begin{aligned}
E(\operatorname{FRAC}(G), t) & =\frac{\delta\left(\mathcal{P}(G), t^{2}\right)+t^{2 d-1} \delta\left(\mathcal{P}(G), 1 / t^{2}\right)}{\left(1-t^{2}\right)^{d+1}} \\
& =\frac{\delta_{0}+\delta_{d-1} t+\delta_{1} t^{2}+\delta_{d-2} t^{3}+\cdots+\delta_{d-1} t^{2 d-2}+\delta_{0} t^{2 d-1}}{\left(1-t^{2}\right)^{d+1}},
\end{aligned}
$$

where $\left(\delta_{0}, \delta_{d-1}, \delta_{1}, \delta_{d-2}, \ldots, \delta_{d-1}, \delta_{0}\right)$ is symmetric and unimodal. In addition,

$$
\begin{aligned}
i^{\text {odd }}(\operatorname{FRAC}(G), 2 k+1) & =(-1)^{d} i^{\text {even }}(\operatorname{FRAC}(G),-2 k-4) \\
& =(-1)^{d} i(\mathcal{P}(G),-k-2) .
\end{aligned}
$$

Proof. Let $W=\operatorname{FRAC}(G)$ and $P=\mathcal{P}(G)$. Then

$$
E(W, t)=\sum_{k \geq 0} i^{\text {even }}(W, 2 k) t^{2 k}+\sum_{k \geq 0} i^{\text {odd }}(W, 2 k+1) t^{2 k+1} .
$$

Since $i^{\text {even }}(W, 2 k)=i(2 W, k)=i(P, k)$, we have

$$
\sum_{k \geq 0} i^{\text {even }}(W, 2 k) t^{2 k}=\sum_{k \geq 0} i(P, k)\left(t^{2}\right)^{k}=\frac{\delta\left(P, t^{2}\right)}{\left(1-t^{2}\right)^{d+1}} .
$$

Since the degree of $i^{\text {odd }}(W, 2 k+1)$ is $d$, by [8, Corollary 4.3.1], we have

$$
\sum_{k \geq 0} i^{\text {odd }}(W, 2 k+1) t^{2 k+1}=t \sum_{k \geq 0} i^{\text {odd }}(W, 2 k+1)\left(t^{2}\right)^{k}=t \frac{a\left(t^{2}\right)}{\left(1-t^{2}\right)^{d+1}}
$$

where $a(t)$ is a polynomial of degree $\leq d$. Thus

$$
E(W, t)=\frac{\delta\left(P, t^{2}\right)}{\left(1-t^{2}\right)^{d+1}}+t \cdot \frac{a\left(t^{2}\right)}{\left(1-t^{2}\right)^{d+1}}=\frac{\delta\left(P, t^{2}\right)+t a\left(t^{2}\right)}{\left(1-t^{2}\right)^{d+1}} .
$$


Since the degree of $E(W, t)$ is -3 as a rational function, the degree of $\delta\left(P, t^{2}\right)+$ $t a\left(t^{2}\right)$ is $2 d-1$. Hence $\operatorname{deg} a(t)=d-1(=\operatorname{deg} \delta(P, t))$. Moreover, since the Ehrhart ring of $W$ is Gorenstein, the coefficients of $\delta\left(P, t^{2}\right)+t a\left(t^{2}\right)$ are symmetric. Thus $a(t)=t^{d-1} \delta(P, 1 / t)$ and $\delta\left(P, t^{2}\right)+t a\left(t^{2}\right)=\delta\left(P, t^{2}\right)+$ $t^{2 d-1} \delta\left(P, 1 / t^{2}\right)$. It is known that $E(P, 1 / t)=-\sum_{k \geq 1} i(P,-k) t^{k}$ (see [9, Chapter 4]). Hence

$$
\begin{aligned}
\sum_{k \geq 0} i^{\text {odd }}(W, 2 k+1) t^{2 k+1} & =\frac{t^{2 d-1} \delta\left(P, 1 / t^{2}\right)}{\left(1-t^{2}\right)^{d+1}} \\
& =\frac{(-1)^{d+1}}{t^{3}} \frac{\delta\left(P, 1 / t^{2}\right)}{\left(1-1 / t^{2}\right)^{d+1}} \\
& =\frac{(-1)^{d+1}}{t^{3}} E\left(P, 1 / t^{2}\right) \\
& =\frac{(-1)^{d}}{t^{3}} \sum_{k \geq 1} i(P,-k) t^{2 k}
\end{aligned}
$$

Thus $i^{\text {odd }}(W, 2 k+1)=(-1)^{d} i(P,-k-2)=(-1)^{d} i^{\text {even }}(W,-2 k-4)$, as desired.

Example 3.2. Let $W=\operatorname{FRAC}\left(K_{d}\right)$ and $P=\mathcal{P}\left(K_{d}\right)$ where $K_{d}$ is a complete graph with $d$ vertices. It is known [13, Example 27] that $\delta(P, t)=A_{d}(t)+$ $d t A_{d-1}(t)$. Let

$$
E(W, t)=\frac{b_{0}+b_{1} t+\cdots+b_{2 d-1} t^{2 d-1}}{\left(1-t^{2}\right)^{d+1}} .
$$

Since

$$
\begin{aligned}
\delta(P, t) & =A_{d}(t)+d t A_{d-1}(t) \\
& =\sum_{i=0}^{d-1} A(d, i) t^{i}+d t \sum_{i=0}^{d-2} A(d-1, i) t^{i} \\
& =\sum_{i=0}^{d-1} A(d, i) t^{i}+d \sum_{i=1}^{d-1} A(d-1, i-1) t^{i} \\
& =1+\sum_{i=1}^{d-1}(A(d, i)+d A(d-1, i-1)) t^{i}
\end{aligned}
$$

hold, the $\delta$-vector $\left(\delta_{0}, \ldots, \delta_{d-1}\right)$ of $P$ satisfies $\delta_{0}=1$ and $\delta_{i}=A(d, i)+d A(d-$ $1, i-1)$ for $i=1,2, \ldots, d-1$. By Theorem 3.1. we can obtain the formula $b_{0}=1$ and $b_{i}=A(d,\lfloor i / 2\rfloor)+d A(d-1,\lfloor(i-1) / 2\rfloor)$ for $i=1,2, \ldots, 2 d-1$.

Example 3.3. Let $W_{d}=\operatorname{FRAC}\left(C_{d}\right)$ where $C_{d}$ is an odd cycle of length $d$. We computed the numerator $g\left(W_{d}, t\right)$ of $E\left(W_{d}, t\right)=g\left(W_{d}, t\right) /\left(1-t^{2}\right)^{d+1}$ for $d=3,5,7,9$ by using software Normaliz ([2]).

$$
g\left(W_{3}, t\right)=1+4 t+7 t^{2}+7 t^{3}+4 t^{4}+t^{5} .
$$




$$
\begin{aligned}
g\left(W_{5}, t\right)= & 1+11 t+51 t^{2}+131 t^{3}+206 t^{4} \\
& +206 t^{5}+131 t^{6}+51 t^{7}+11 t^{8}+t^{9} . \\
g\left(W_{7}, t\right)= & 1+29 t+281 t^{2}+1408 t^{3}+4320 t^{4}+8814 t^{5}+12475 t^{6} \\
& +12475 t^{7}+8814 t^{8}+4320 t^{9}+1408 t^{10}+281 t^{11}+29 t^{12}+t^{13} . \\
g\left(W_{9}, t\right)= & 1+76 t+1450 t^{2}+12844 t^{3}+67000 t^{4}+230986 t^{5}+561004 t^{6} \\
& +996310 t^{7}+1321369 t^{8}+1321369 t^{9}+996310 t^{10}+561004 t^{11} \\
& +230986 t^{12}+67000 t^{13}+12844 t^{14}+1450 t^{15}+76 t^{16}+t^{17} .
\end{aligned}
$$

\section{The dual polytope of $\mathcal{Q}(G)$}

In this section, we will discuss the dual polytope $\mathcal{Q}(G)^{\vee}$ of $\mathcal{Q}(G)$. Recall that

$$
\mathcal{Q}(G)^{\vee}=\operatorname{Conv}\left(\left\{\mathbf{e}_{i}+\mathbf{e}_{j} \mid(i, j) \in E(G)\right\} \cup\left\{-\mathbf{e}_{i} \mid 1 \leq i \leq d\right\}\right)
$$

if $G$ has no isolated vertices. It is easy to see that $\mathcal{Q}(G)^{\vee}$ is Fano. A lattice polytope $P \subset \mathbb{R}^{d}$ is called normal if $\mathbb{Z}_{\geq 0} A=\mathbb{Q}_{\geq 0} A \cap \mathbb{Z} A$, where

$$
A=\left(\begin{array}{ccc}
\mathbf{a}_{1} & \cdots & \mathbf{a}_{n} \\
1 & \cdots & 1
\end{array}\right)
$$

such that $\left\{\mathbf{a}_{1}, \ldots, \mathbf{a}_{n}\right\}=P \cap \mathbb{Z}^{d}$. Here $\mathbb{Z} A=\left\{\sum_{i=1}^{n} z_{i}\left(\mathbf{a}_{i}, 1\right) \mid z_{i} \in \mathbb{Z}\right\}$, for example. A triangulation $\Delta$ of $P$ is called unimodular if the normalized volume of each maximal simplex of $\Delta$ is one. If $\mathbb{Z} A=\mathbb{Z}^{d+1}$, then the normalized volume of each maximal simplex is equal to the absolute value of the corresponding maximal minor of $A$. See, 44, Section 5.5]. It is known that a lattice polytope $P$ is normal if $P$ has a unimodular triangulation (4, Theorem 5.6.7]).

Theorem 4.1. Let $G$ be a finite simple graph without isolated vertices. Then the following conditions are equivalent.

(i) The graph $G$ is a bipartite graph;

(ii) The dual polytope $\mathcal{Q}(G)^{\vee}$ has a unimodular triangulation;

(iii) The dual polytope $\mathcal{Q}(G)^{\vee}$ is normal;

(iv) The dual polytope $\mathcal{Q}(G)^{\vee}$ is a Gorenstein Fano polytope.

Proof. Since $\mathcal{Q}(G)^{\vee}$ is Fano, and since $\left(\mathcal{Q}(G)^{\vee}\right)^{\vee}=\mathcal{Q}(G), \mathcal{Q}(G)^{\vee}$ is Gorenstein Fano if and only if $\mathcal{Q}(G)$ is a lattice polytope. By Proposition 2.3, $\mathcal{Q}(G)$ is a lattice polytope if and only if $G$ is bipartite. Hence we have (i) $\Leftrightarrow$ (iv). Moreover, (ii) $\Rightarrow$ (iii) holds in general. Let $\mathcal{A}_{G}$ be the vertex-edge incidence matrix of $G$ and let $\mathcal{A}_{G}^{\prime}$ be the configuration matrix of $\mathcal{Q}(G)^{\vee}$, namely,

$$
\mathcal{A}_{G}^{\prime}=\left(\begin{array}{ccc}
\mathbf{0} & \mathcal{A}_{G} & -E_{d} \\
1 & 1 \cdots 1 & 1 \cdots 1
\end{array}\right),
$$

where $E_{d}$ is an identity matrix. Then $\mathbb{Z} \mathcal{A}_{G}^{\prime}=\mathbb{Z}^{d+1}$. Hence $\mathcal{Q}(G)^{\vee}$ is normal if and only if $\mathbb{Z}_{\geq 0} \mathcal{A}_{G}^{\prime}=\mathbb{Q}_{\geq 0} \mathcal{A}_{G}^{\prime} \cap \mathbb{Z}^{d+1}$.

(i) $\Rightarrow$ (ii): Suppose that $G$ is bipartite. It is known [11 that the vertexedge incidence matrix of any bipartite graph is totally unimodular, i.e., the 
determinant of every square non-singular submatrix is \pm 1 . Hence it follows that the submatrix $B=\left(\begin{array}{ll}\mathcal{A}_{G} & -E_{d}\end{array}\right)$ of $\mathcal{A}_{G}^{\prime}$ is totally unimodular. Let $\Delta$ be a pulling triangulation ([1], [4, Proposition 5.6.5]) of $\mathcal{Q}(G)^{\vee}$ such that the origin is a vertex of every maximal simplex in $\Delta$. Such a triangulation is obtained by a Gröbner basis of the toric ideal of $\mathcal{A}_{G}^{\prime}$ with respect to a reverse lexicographic order such that the smallest variable corresponds to the origin. Then the normalized volume of each maximal simplex in $\Delta$ is equal to the absolute value of the corresponding maximal minor of $B$. Since $B$ is totally unimodular, each maximal minor of $B$ is \pm 1 , and hence the triangulation $\Delta$ is unimodular.

(iii) $\Rightarrow$ (i): Suppose that the graph $G$ contains an odd cycle $C$. Now, we will show that $\mathcal{Q}(G)^{\vee}$ is not normal, that is, $\mathbb{Z}_{\geq 0} \mathcal{A}_{G}^{\prime} \neq \mathbb{Q}_{\geq 0} \mathcal{A}_{G}^{\prime} \cap \mathbb{Z}^{d+1}$. We may assume that $C=(1,2, \ldots, 2 k+1)$. Let

$$
\begin{aligned}
\mathbf{u} & =\frac{1}{2}\left(\mathbf{e}_{d+1}+\left(\mathbf{e}_{1}+\mathbf{e}_{2 k+1}+\mathbf{e}_{d+1}\right)+\sum_{i=1}^{2 k}\left(\mathbf{e}_{i}+\mathbf{e}_{i+1}+\mathbf{e}_{d+1}\right)\right) \\
& =(k+1) \mathbf{e}_{d+1}+\sum_{i=1}^{2 k+1} \mathbf{e}_{i} .
\end{aligned}
$$

Then $\mathbf{u}$ belongs to $\mathbb{Q}_{\geq 0} \mathcal{A}_{G}^{\prime} \cap \mathbb{Z}^{d+1}$. It is enough to show that $\mathbf{u} \notin \mathbb{Z}_{\geq 0} \mathcal{A}_{G}^{\prime}$. Suppose

$$
\mathbf{u}=\gamma \mathbf{e}_{d+1}+\sum_{(i, j) \in E(G)} \alpha_{i j}\left(\mathbf{e}_{i}+\mathbf{e}_{j}+\mathbf{e}_{d+1}\right)+\sum_{i=1}^{d} \beta_{i}\left(-\mathbf{e}_{i}+\mathbf{e}_{d+1}\right)
$$

for some $\alpha_{i j}, \beta_{i} \in \mathbb{Z}_{\geq 0}$. Then the coefficient of $\mathbf{e}_{i}(1 \leq i \leq 2 k+1)$ in (1) is $1=\sum_{(i, j) \in E(G)} \alpha_{i j}-\beta_{i}$, and that of $\mathbf{e}_{i}(2 k+2 \leq i \leq d)$ in (11) is $0=$ $\sum_{(i, j) \in E(G)} \alpha_{i j}-\beta_{i}$. By summing up the equations for $1 \leq i \leq d$, we obtain

$$
2 k+1=2 \sum_{(i, j) \in E(G)} \alpha_{i j}-\sum_{i=1}^{d} \beta_{i} .
$$

On the other hand, the coefficient of $\mathbf{e}_{d+1}$ in (1) is

$$
k+1=\gamma+\sum_{(i, j) \in E(G)} \alpha_{i j}+\sum_{i=1}^{d} \beta_{i} .
$$

Since $\gamma$ and $\sum_{i=1}^{d} \beta_{i}$ are nonnegative, by equations (2) and (3), we obtain

$$
k+\frac{1}{2} \leq \sum_{(i, j) \in E(G)} \alpha_{i j} \leq k+1 .
$$

Since $\sum_{(i, j) \in E(G)} \alpha_{i j} \in \mathbb{Z}$, it follows that $\sum_{(i, j) \in E(G)} \alpha_{i j}=k+1$. Hence, by equation (2), we have $\sum_{i=1}^{d} \beta_{i}=1$. Thus, by equation (3), we have $\gamma+1=0$, which is a contradiction. 


\title{
References
}

[1] C. A. Athanasiadis, $h^{*}$-vectors, Eulerian polynomials and stable polytopes of graphs, Electron. J. Combin. 11 (2004/06), no. 2, Research Paper 6, 13 pp. (electronic).

[2] W. Bruns, B. Ichim, T. Römer, R. Sieg and C. Söger, Normaliz. Algorithms for rational cones and affine monoids.

Available at https://www.normaliz.uni-osnabrueck.de.

[3] W. Bruns and T. Römer, $h$-Vectors of Gorenstein polytopes, J. Combin. Theory Ser. A 114 (2007), 65-76.

[4] T. Hibi, Ed., "Gröbner Bases: Statistics and Software Systems," Springer, 2013.

[5] E. De Negri and T. Hibi, Gorenstein algebras of Veronese type, J. Algebra 193 (1997), 629-639.

[6] G. L. Nemhauser and L. E. Trotter, Jr., Properties of vertex packing and independence system polyhedra, Math. Programming 6 (1974), 48-61.

[7] H. Ohsugi and T. Hibi, Special simplices and Gorenstein toric rings, J. Combin. Theory Ser. A 113 (2006), 718-725.

[8] R. P. Stanley, "Enumerative Combinatorics" Volume 1 second edition, Wadsworth \& Brook, Monterey, Wadsworth \& Brooks/Cole Math Series, 1986.

[9] R. P. Stanley, Two poset polytopes, Discrete Comput. Geom. 1 (1986), 9-23.

[10] J. Schepers and L. Van Langenhoven, Unimodality questions for integrally closed lattice polytopes, Ann. Comb. 17 (2013), 571-589.

[11] A. Schrijver, "Theory of Linear and Integer Programming," John Wiley \& Sons, Ltd., Chichester, 1986.

[12] A. Stapledon, Inequalities and Ehrhart $\delta$-vectors, Trans. Amer. Math. Soc. 361 (2009), 5615-5626.

[13] E. Steingrímsson, A decomposition of 2-weak vertex-packing polytopes, Discrete Comput. Geom. 12 (1994), 465-479.

\author{
Ginji Hamano \\ Department of Pure and Applied Mathematics \\ Graduate School of Information Science and Technology \\ Osaka University \\ Suita, Osaka 565-0871 \\ Japan \\ e-mail: g-hamano@ist.osaka-u.ac.jp \\ Takayuki Hibi \\ Department of Pure and Applied Mathematics \\ Graduate School of Information Science and Technology \\ Osaka University \\ Suita, Osaka 565-0871 \\ Japan \\ e-mail: hibi@math.sci.osaka-u.ac.jp
}




\author{
Hidefumi Ohsugi \\ Department of Mathematical Sciences \\ School of Science and Technology \\ Kwansei Gakuin University \\ Sanda, Hyogo 669-1337 \\ Japan \\ e-mail: ohsugi@kwansei.ac.jp
}

